\title{
RELAÇÃO ENTRE ÓXIDOS DE FERRO E DE MANGANÊS E A SORÇÃO DE FÓSFORO EM SOLOS NO RIO GRANDE DO SUL ${ }^{(1)}$
}

\author{
Gustavo Kruger Gonçalves ${ }^{(2)}$, Egon José Meurer ${ }^{(3)}$, Leandro \\ Bortolon $^{(4)}$ \& Daiana Ribeiro Nunes Gonçalves ${ }^{(5)}$
}

\begin{abstract}
RESUMO
Os solos utilizados para a cultura de arroz irrigado por alagamento no Estado do Rio Grande do Sul são oriundos de diferentes materiais de origem e estão sujeitos às variações temporais das condições de oxidação e redução. Essas condições contribuem para as variações nos teores totais e na crista-linidade de óxidos de Fe e de Mn, as quais estão relacionadas aos processos de sorção e dessorção, afetando a disponibilidade de $\mathbf{P}$ para a cultura de arroz irrigado. Com o objetivo de verificar a sorção de $\mathrm{P}$ e a sua disponibilidade para o arroz em solos oriundos de diferentes materiais de origem, foram desenvolvidos dois estudos: o primeiro para determinar os teores totais de Fe e de Mn e a capacidade máxima de adsorção de P (CMAP) pelas isotermas de Langmuir; além disso, foram associados a CMAP e os teores totais de $\mathrm{Fe}$ e de $\mathrm{Mn}$, na razão $\mathrm{Fe}_{\mathrm{Ox} 6} / \mathrm{Fe}_{\text {dit }}$, razão $\mathrm{Mn}_{\mathrm{Ox} 6} / \mathrm{Mn}_{\mathrm{dit}}$ argila e da matéria orgânica. $O$ segundo estudo foi para quantificar a sorção de $P$ durante quatro períodos de alagamento do solo. Os solos oriundos de sedimento de basalto (Luvissolo, Vertissolo e Chernossolo) apresentaram maior CMAP do que os oriundos de sedimento de granito e arenito (Planossolos e Gleissolos), devido aos maiores teores totais de Fe e também às razões de Fe extraído por oxalato de amônio a pH 6 $\left(\mathrm{Fe}_{\mathrm{ox}}\right)$ /ferro extraído por ditionito $\left(\mathrm{Fe}_{\mathrm{dit}}\right)$ e $\mathrm{Mn}$ extraído por oxalato de amônio a pH $6\left(\mathrm{Mn}_{\mathrm{ox} 6}\right) / \mathrm{Mn}_{\text {dit }}$. A razão $\mathrm{Fe}_{\mathrm{ox} 6} / \mathrm{Fe}_{\text {dit }}$ apresentou a maior associação com a CMAP em todos os solos utilizados. O Planossolo teve menor sorção de P do que os solos oriundos de sedimento de basalto em todos os períodos de alagamento.
\end{abstract}

Termos de indexação: material de origem, oxidação, redução, solução do solo e nutrientes.

\footnotetext{
(1) Parte da tese de Doutorado em Ciência do Solo do primeiro autor apresentada à Universidade Federal do Rio Grande do Sul - UFRGS. Recebido para publicação em 18 de agosto de 2010 e aprovado em 27 de julho de 2011.

(2) Professor da Universidade Estadual do Rio Grande do Sul - UERGS. E-mail: gustavo-goncalves@uergs.edu.br

(3) Professor Associado do Departamento de Solos, UFRGS. E-mail: egon.meurer@ufrgs.br

(4) Doutor em Ciência do Solo, UFRGS. E-mail: leandro.bortolon@ufrgs.br

(5) Mestre em Agronomia, Universidade Federal de Pelotas - UFPEL.
} 


\title{
SUMMARY: RELATIONSHIP BETWEEN IRON AND MANGANESE OXIDES AND PHOSPHORUS SORPTION IN SOILS IN THE STATE OF RIO GRANDE DO SUL, BRAZIL
}

\begin{abstract}
The soils used for irrigated rice by flooding in the state of Rio Grande do Sul, Brazil, are derived from different source material and are subjected to temporal variations in oxidation and reduction conditions. These conditions contribute to variations in total concentrations and crystallinity of iron oxides and manganese, which are related to sorption and desorption processes, affecting $P$ availability to irrigated rice. With the objective of verifying $P$ sorption and availability to rice in soils from different parent materials, two studies were conducted. In the first, the total content of Fe and Mn and maximum Padsorption capacity (MPAC) by the Langmuir Isotherm were determined, and associated with the MPAC, the total contents of Fe and $\mathrm{Mn}, \mathrm{Fe}_{\mathrm{Ox} 6} / \mathrm{Fe}_{\text {dit }}$ ratio $\mathrm{Mn} n_{\mathrm{Ox} 6} / \mathrm{Mn}_{\text {dit }}$ ratio, clay and organic matter. In the second study, $P$ sorption was quantified in four flooding periods. In the soils derived from basalt sediment (Luvisolo, Vertisolo and Chernosolo), MPAC was higher than in soils derived from granite and sandstone sediment (Planosolos and Gleisolos), due to higher total of Fe and Mn contents and $\mathrm{FeOx}_{6} / \mathrm{Fe}_{\text {dit }}$ and $\mathrm{FeO} x_{3} / \mathrm{Fe}_{\text {dit }}$ ratios. The association with $\mathrm{MPAC}$ was highest with $\mathrm{FeOx} x_{6} / \mathrm{Fe}_{\text {dit }}$ in all soils. P sorption was lower in the Planossolo than in soils derived from basalt sediment in all flooding periods.
\end{abstract}

Index terms: parent material, oxidation, reduction, soil solution and nutrients.

\section{INTRODUÇÃO}

Os solos de várzea do Rio Grande do Sul são utilizados, principalmente, para o cultivo de arroz irrigado por alagamento. São oriundos de diferentes materiais de origem (basalto, arenito, siltito e granito), os quais contribuem para variações nos teores totais de óxidos de Fe e de Mn. As variações temporais das condições de oxidação e redução dos solos afetam a sua cristalinidade, influenciando nas diferentes taxas de solubilização de $\mathrm{Fe}$ e, indiretamente, nas concentrações de P na solução dos solos reduzidos. As formas de Fe atuam na sorção de $\mathrm{P}$, seja em ambientes oxidados ou em ambientes reduzidos, nas seguintes situações: (a) anteriormente ao processo de redução dos óxidos férricos a óxidos ferrosos, principalmente em solos com maiores teores de óxidos de ferro cristalinos $\left(\mathrm{Fe}_{\mathrm{dit}}\right)$ e de baixa cristalinidade $\left(\mathrm{Fe}_{\mathrm{ox}}\right)$, os quais apresentam ponto de carga zero (PCZ) entre $7 \mathrm{e}$ 9, resultando em cargas superficiais positivas, favorecendo a sorção de P (Schwertmann \& Taylor, 1989); (b) durante o processo de redução dos óxidos férricos a óxidos ferrosos, devido ao aumento das concentrações de $\mathrm{P}$ e Fe na solução, proporcionando a precipitação de fosfatos de ferro. Da mesma maneira, o aumento do $\mathrm{pH}$ diminui a solubilidade dos óxidos de Fe de baixa cristalinidade, os quais precipitam, elevando a capacidade de adsorção de $\mathrm{P}$ (Khalid et al., 1977; Ponnamperuma, 1978; Sah et al., 1989).

Em relação aos óxidos de Mn, alguns trabalhos têm evidenciado resultados contraditórios, já que em solos com predominância de óxidos de Mn de baixa cristalinidade $\left(\mathrm{Mn}_{\mathrm{ox}}\right)$ os óxidos mangânicos podem, primeiramente, adsorver o $\mathrm{P}$ (Shahandeh et al., 2003). Essa adsorção pelos óxidos de Mn é favorecida quando o PCZ desse elemento for superior ao $\mathrm{pH}$ do solo. Os óxidos de Mn apresentam PCZ entre 1,5 e 7,5, e a birnessita de baixa cristalinidade, que é a forma mais comum de ocorrência desses óxidos, apresenta PCZ de 1,5 (Mckenzie, 1989). Logo, não é de se esperar que ela influencie na sorção e dessorção de P.

A capacidade máxima de adsorção de fósforo (CMAP) descrita pela Isoterma de Langmuir em solos oxidados e os teores de óxidos de Fe e de Mn cristalinos e de baixa cristalinidade podem ser utilizados para explicar a adsorção de $\mathrm{P}$ que ocorre em solo oxidado anteriormente à redução dos óxidos férricos e mangânicos. Além disso, é necessário quantificar a sorção de $\mathrm{P}$ durante o processo de redução em solos oriundos de diferentes materiais de origem. Sousa (2001) observou que um Plintossolo, oriundo de sedimento de basalto, apresentou cinética diferenciada de solubilização de $\mathrm{Mn}$ e $\mathrm{Fe}$ em relação a um Planossolo, enquanto Gonçalves et al. (2008) observaram que um Cambissolo, oriundo de sedimento de basalto, mostrou cinética diferenciada de $\mathrm{P}$ em relação a um Planossolo. Logo, deve ser quantificada a adsorção de $\mathrm{P}$ em diferentes períodos de alagamento em solos oriundos de distintos materiais, objetivando confirmar as prováveis diferenças observadas naqueles estudos. Ambas as quantificações serão importantes para predizer a disponibilidade de $P$ para a cultura de arroz irrigado, bem como para as culturas de inverno, que sucedem a lavoura de arroz irrigado.

Este trabalho foi realizado partindo-se das seguintes hipóteses: (a) Os solos oriundos de sedimento de basalto têm maiores teores totais de $\mathrm{Fe}$, proporcionando maior CMAP do que os solos derivados de sedimento de arenito e granito; (b) A CMAP tem maior associação com a razão $\mathrm{Fe}_{\mathrm{Ox} 6} / \mathrm{Fe}_{\text {dit }}$ do que com a razão $\mathrm{Mn}_{\mathrm{Ox} 6} /$ 
$\mathrm{Mn}_{\text {dit. }}$ e (c) Os solos oriundos de sedimento de basalto têm maiores teores de óxidos de $\mathrm{Mn}$ e de Fe, proporcionando cinética de sorção de $\mathrm{P}$ diferenciada em relação ao solo oriundo de sedimento de granito durante o período de alagamento. Teve também os seguintes objetivos: quantificar os teores totais de $\mathrm{Fe}$ e de Mn e as razões entre os óxidos de baixa cristalinidade e os óxidos cristalinos; estimar a CMAP pela isoterma de Langmuir; associar a CMAP e os teores totais de Fe e de Mn, bem como as razões entre os óxidos de baixa cristalinidade e os óxidos cristalinos; e quantificar a sorção de $\mathrm{P}$ durante quatro períodos de alagamento do solo.

\section{MATERIAL E MÉTODOS}

Foram selecionados os solos mais representativos utilizados para o cultivo do arroz irrigado no Estado do Rio Grande do Sul: (a) Planossolo hidromórfico eutrófico solódico (SGe 3), coletado no município de Pelotas; (b) Gleissolo háplico Ta distrófico (GXd), coletado no município de Cachoeirinha; (c) Planossolo hidromórfico eutrófico arênico (SGe 1), coletado no município de Restinga Seca; (d) Luvissolo crômico pálico abrúptico (TCp), coletado no município de Itaqui; e (e) Vertissolo ebânico órtico típico coletado (VEo 2) e Chernossolo ebânico carbonático vértico (MEk), ambos coletados no município de Alegrete. Após a coleta, as amostras superficiais $(0-20 \mathrm{~cm})$ foram caracterizadas quimicamente, segundo método (Quadro 1) descritos em Tedesco et al. (1995).

\section{Caracterização das frações de ferro e de manganês}

Os óxidos de ferro $\left(\mathrm{Fe}_{\mathrm{dit}}\right)$ e de manganês $\left(\mathrm{Mn}_{\mathrm{dit}}\right) \mathrm{de}$ elevada cristalinidade foram extraídos com a utilização de três sais: ditionito de sódio, citrato de sódio e bicarbonato de sódio a $80^{\circ} \mathrm{C}$, em duas extrações sucessivas (Mehra \& Jackson, 1960; Inda Junior \& Kampf, 2003). Em cada extração de $15 \mathrm{~min}$, adicionou-se $1 \mathrm{~g}$ de ditionito de sódio, parcelado em três alíquotas de aproximadamente $0,33 \mathrm{~g}$, a 0,2 g de terra fina seca ao ar (TFSA) contida em $40 \mathrm{~mL}$ de solução de citrato de sódio $0,3 \mathrm{~mol} \mathrm{~L}^{-1}$ : bicarbonato de sódio $1 \mathrm{~mol} \mathrm{~L}^{-1}$, na razão 8:1, em tubo de centrífuga com capacidade para $50 \mathrm{~mL}$. Após a adição de cada alíquota de ditionito de sódio, a suspensão foi agitada por $1 \mathrm{~min}$. A temperatura de extração foi mantida a $80^{\circ} \mathrm{C}$ em banho-maria. Ao fim de cada etapa de extração, a suspensão foi centrifugada a $3.000 \mathrm{rpm}$ por 10 min e o extrato, completado para $50 \mathrm{~mL}$ com a solução citrato-bicarbonato.

Os óxidos de Fe e de Mn de baixa cristalinidade $\left(\mathrm{Fe}_{\mathrm{Ox}}, \mathrm{Mn}_{\mathrm{Ox}}\right)$ foram extraídos com oxalato de amônio $\left[\left(\left(\mathrm{NH}_{4}\right)_{2} \mathrm{C}_{2} \mathrm{O}_{4} \cdot \mathrm{H}_{2} \mathrm{O}\right)\right]$ 0,2 $\mathrm{mol} \mathrm{L}^{-1}$ a pH 3,0 $\left(\mathrm{Fe}_{\text {ox } 3}\right)$ e pH 6,0 $\left(\mathrm{Fe}_{\text {ox } 6}\right)$, no escuro. Anteriormente à extração, foi realizada a correção de $\mathrm{pH}$ na solução de oxalato de amônio, conforme Schwertmann (1964). Posteriormente, foi realizada a única extração, em que 0,2 g de TFSA e $40 \mathrm{~mL}$ de solução de oxalato de amônio foram adicionados em tubo plástico de $50 \mathrm{~mL}$ de volume, recobertos com papel-alumínio para evitar a incidência direta de luz na solução e fechados com tampas. As suspensões foram agitadas por $2 \mathrm{~h}$ em agitador horizontal e, ao final, centrifugadas a $2.000 \mathrm{rpm}$ por $5 \mathrm{~min}$. O sobrenadante coletado foi completado com oxalato de amônio a $50 \mathrm{~mL}$, em balão volumétrico.

As dissoluções seletivas dos óxidos de Fe e de Mn foram realizadas em triplicata e os teores de Fe e de Mn solubilizados pelos dois extratores, determinados por espectrometria de absorção atômica (EAA).

\section{Avaliação da adsorção de $\mathbf{P}$ em solo oxidado}

Para determinação do $\mathrm{P}$ em condições oxidadas, foram realizados os seguintes procedimentos: em frascos de snap-cap de $100 \mathrm{~mL}$, equilibraram-se duplicatas de $5 \mathrm{~g}$ de solo com $50 \mathrm{~mL}$ de uma solução de $\mathrm{KCl}$ 0,02 $\mathrm{mol} \mathrm{L}^{-1}$, com concentrações variáveis de $\mathrm{P}$ adicionado como $\mathrm{K}\left(\mathrm{H}_{2} \mathrm{PO}_{4}\right)$. As concentrações de $\mathrm{P}$ adicionadas às soluções foram: $10,15,25,50,100$, 200,300 e $400 \mathrm{mg} \mathrm{L}^{-1}$, exceto as amostras de Planossolo, que receberam as soluções de 2,$5 ; 5 ; 10$; $15 ; 25 ; 50 ; 100 ; 150 ; 200 ; 300$; e $400 \mathrm{mg} \mathrm{L}^{-1}$. Nos solos da classe Planossolo foram adicionadas as oito primeiras soluções, enquanto nos outros solos, as demais soluções. Em seguida, as amostras dos solos foram equilibradas com as soluções, com agitação horizontal por $2 \mathrm{~h}$ (100-120 oscilações por minuto). Posteriormente, foram afrouxadas as tampas, deixando-as em repouso até o dia seguinte $(15 \mathrm{~h})$. Depois desse período, foram transferidos $3 \mathrm{~mL}$ do sobrenadante para um copo plástico, em que foram adicionados $3 \mathrm{~mL}$ de solução de $\mathrm{KCl} 0,02 \mathrm{~mol} \mathrm{~L}^{-1}$, agitados levemente e acrescidos de solução redutora de molibdato de amônio e ácido ascórbico (Murphy \& Rilley, 1962). Em seguida, foi determinada a absorbância no espectrofotômetro de absorção a $882 \mathrm{~nm}$.

O P adsorvido na fase sólida foi calculado por diferença entre o $\mathrm{P}$ adicionado e o quantificado na solução de equilíbrio. Os valores obtidos de cada solo foram ajustados à equação de Langmuir, descrita a seguir:

$$
\mathrm{x} / \mathrm{m}=\mathrm{kbc} /(1+\mathrm{kc})
$$

em que: $\mathrm{x} / \mathrm{m}=$ quantidade de $\mathrm{P}$ adsorvido por unidade de massa de solo; $\mathrm{k}=$ constante relacionada com energia de ligação; $b$ = capacidade máxima de adsorção de P; e c = concentração de P na solução em equilíbrio.

Para a estimativa da constante $b$, a equação 1 foi transformada em sua forma linear:

$$
(y=b 0+b 1 x): x / m=1 /(k b)+(1 / b) c
$$

O valor da CMAP(b) foi estimado a partir da declividade da reta ajustada b1. Dessa forma, CMAP $=1 / \mathrm{b} 1$. 
Foram feitas análises de correlação linear simples entre as variáveis e verificado o grau de associação entre estas pelo valor do coeficiente de correlação ( $\mathrm{p}<0,05)$, utilizando-se o Sistema de Análise Estatística para Microcomputadores -SANEST (Zonta et al., 1984).

\section{Avaliação da adsorção de $\mathrm{P}$ em solo reduzido}

Para determinação do $\mathrm{P}$ em condições reduzidas, foi utilizado o delineamento em blocos ao acaso com três repetições. O experimento adotado foi um fatorial $4 \mathrm{x} 4$, em que foram utilizados os seguintes fatores e respectivos níveis: (a) Solos: Planossolo (SGe 3), Luvissolo (TCp), Vertissolo (VEo 2) e Chernossolo (MEk); e (b) Períodos de alagamento: 5, 15, 30 e 45 dias de alagamento.

Amostras de $50 \mathrm{~g}$ de solo foram adicionadas em snap-cap de $200 \mathrm{~mL}$ (unidades experimentais) e posteriormente receberam adição de $50 \mathrm{~mL}$ de água destilada referentes ao tratamento-testemunha, enquanto as demais unidades receberam $25 \mathrm{~mL}$ de água destilada e $25 \mathrm{~mL}$ da solução de $\mathrm{KH}_{2} \mathrm{PO}_{4}$ com concentração de $400 \mathrm{mg} \mathrm{L}^{-1} \mathrm{P}$, resultando em $200 \mathrm{mg} \mathrm{L}^{-1} \mathrm{P}$. As unidades experimentais foram colocadas em estufa com temperatura de aproximadamente $30^{\circ}$, sendo cobertas com plástico preto e tampadas, para evitar o crescimento de algas e também a troca de gases. Aos 5, 15, 30 e 45 dias de alagamento, retirou-se uma alíquota para obtenção dos teores de $\mathrm{P}$ e de Fe na solução. O P adsorvido foi obtido de acordo com a equação 3 , sendo o $\mathrm{P}$ presente na solução dos tratamentos que receberam a adição da fonte fosfatada subtraído do P quantificado na solução dos tratamentos que não a receberam, o que corresponde ao $\mathrm{P}$ nativo liberado com a redução do solo. Ou seja:

$\mathrm{P}$ adsorvido $\left(\mathrm{mg} \mathrm{kg}^{-1}\right)=\mathrm{P}$ adicionado $\left(\mathrm{mg} \mathrm{kg}^{-1}\right)-\mathrm{P}$ solução do tratamento com adição de $\mathrm{P}\left(\mathrm{mg} \mathrm{kg}^{-1}\right)$ - $\mathrm{P}$ solução do tratamento sem adição de $\mathrm{P}\left(\mathrm{mg} \mathrm{kg}^{-1}\right)$
Os dados de $\mathrm{P}$ sorvido, Fe, pH e Mn na solução do solo foram submetidos à análise de variância e a comparação de médias, feita pelo teste de Duncan a $5 \%$, utilizando o SANEST.

\section{RESULTADOS E DISCUSSÃO}

Os solos oriundos de sedimento de basalto (Luvissolo, Vertissolo e Chernossolo) apresentaram maiores teores totais de $\mathrm{Fe}$ e também das razões $\mathrm{Fe}_{\text {ox6 }} /$ $\mathrm{Fe}_{\text {dit }}$, quando comparados aos solos oriundos de sedimento de arenito e de granito, representados pelos Planossolos e Gleissolos (Quadro 2). Entre os solos utilizados, o Chernossolo e o Vertissolo apresentaram os maiores teores totais de $\mathrm{Fe}$ e razões $\mathrm{Fe}_{\text {ox } 6} / \mathrm{Fe}_{\text {dit }}$ em relação aos solos oriundos de sedimento de arenito e granito. A maior quantidade de matéria orgânica presente nesses solos indica que eles possuem maior quantidade de ligantes orgânicos, como ácidos carboxílicos, os quais complexam o Fe na superfície da ferridrita, diminuindo a cristalização (Schwertmann, 1966).

Resultados semelhantes foram obtidos por Vahl et al. (1999), os quais observaram maiores teores totais de $\mathrm{Fe}$ e razões $\mathrm{Fe}_{\mathrm{ox} 6} / \mathrm{Fe}_{\text {dit }}$ nos solos Vertissolos e Chernossolos quando comparados aos solos da classe Planossolo e Gleissolo.

$\mathrm{O}$ extrator $\mathrm{Fe}_{\mathrm{ox} 6}$ extraiu menores quantidades de Fe que o extrator $\mathrm{Fe}_{\mathrm{ox} 3}$ (Quadro 2). Isso indica que no valor de pH 6 as condições de solubilização e, ou, complexação de Fe são menores (Hernandez \& Meurer, 1998). Vahl et al. (1993) adaptaram o metodo descrito em Schwertmann (1964) ao valor de $\mathrm{pH} 6$ para estimar, em amostras secas, o conteúdo de Fe que foi reduzido após o alagamento do solo na cultura de arroz irrigado.

A partir do ajuste linear das isotermas de Langmuir, foram indicados os valores da CMAP, os quais variaram de 73 a $588 \mathrm{mg} \mathrm{kg}^{-1}$ (Quadro 3). A maior CMAP observada nos solos oriundos de sedimentos de basalto é atribuída, principalmente, aos maiores teores totais de $\mathrm{Fe}$ e razões $\mathrm{Fe}_{\text {ox } 6} / \mathrm{Fe}_{\text {dit }}$ e $\mathrm{Fe}_{\text {ox } 3} /$

\section{Quadro 1. Atributos das amostras dos solos utilizados no experimento}

\begin{tabular}{|c|c|c|c|c|c|c|c|c|c|c|}
\hline \multirow[t]{2}{*}{$\begin{array}{l}\text { Classificação taxonômica } \\
\text { (Embrapa, 2006) }\end{array}$} & \multirow[t]{2}{*}{$\begin{array}{l}\text { Material } \\
\text { de } \\
\text { origem }\end{array}$} & \multicolumn{3}{|c|}{$\operatorname{Argila}^{(1)} \mathrm{MC}$} & \multirow{2}{*}{$\begin{array}{c}\mathbf{K}^{(3)} \mathbf{P}^{(3)} \\
-\mathrm{mg} \mathrm{kg}^{-1}-\end{array}$} & \multicolumn{2}{|c|}{$F \mathbf{e}_{\mathrm{ox} 6}^{(4)} \mathbf{F} \mathbf{e}_{\mathrm{dit}}^{\left({ }^{(5)}\right.}$} & $\mathrm{Mn}_{\mathrm{ox6}}{ }^{(4)}$ & \multirow{2}{*}{$\mathrm{Mn}_{\mathrm{dit}}^{(5)}$} & \multirow{2}{*}{$\frac{\mathbf{C M A P}^{(6)}}{\mathrm{mg} \mathrm{kg}^{-1}}$} \\
\hline & & $-\mathrm{g}$ & $g^{-1}-$ & & & & g l & $\mathrm{rg}^{-1}$ & & \\
\hline Planossolo háplico eutrófico solódico (SGe3) & Granito & 200 & 22 & 4,7 & 613,0 & 0,37 & 5,50 & 0,12 & 1,65 & 132 \\
\hline Gleissolo háplico Ta distrófico (GXd) & Granito & 220 & 24 & 4,4 & 855,5 & 0,28 & 4,8 & 0,09 & 1,80 & 99 \\
\hline Planossolo háplico eutrófico arênico (SGe1) & Arenito & 120 & 14 & 5,3 & 7515,0 & 0,19 & 3,10 & 0,18 & 2,00 & 73 \\
\hline Luvissolo crômico pálico abrúptico (TCp) & Basalto & 230 & 17 & 4,3 & $80 \quad 2,5$ & 0,92 & 8,00 & 0,36 & 2,20 & 455 \\
\hline Vertissolo ebânico órtico típico (VEo2) & Basalto & 320 & 43 & 5,5 & $102 \quad 2,9$ & 1,35 & 10,70 & 0,49 & 2,20 & 556 \\
\hline Chernossolo ebânico carbonático vértico (MEk) & Basalto & 280 & 26 & 4,9 & $114 \quad 2,7$ & 1,45 & 10,80 & 0,70 & 2,60 & 588 \\
\hline
\end{tabular}

(1) Método de densímetro. ${ }^{(2)}$ Digestão úmida. ${ }^{(3)}$ Método Mehlich-1. ${ }^{(4)}$ Extração com oxalato de amônio. ${ }^{(5)}$ Extração com ditionitocitrato-bicarbonato. ${ }^{(6)}$ Isoterma de Longmuir. 
Quadro 2. Teores totais de Fe e de Mn e as razões entre os óxidos de baixa cristalinidade e cristalinos

\begin{tabular}{|c|c|c|c|c|c|c|c|c|c|c|}
\hline $\begin{array}{l}\text { Classificação taxônomica } \\
\text { Embrapa - CNPS (2006) }\end{array}$ & $\mathrm{Fe}_{\mathrm{ox} 6}$ & $\mathrm{Fe}_{\mathrm{ox} 3}$ & $\mathrm{Fe}_{\text {dit }}$ & $\begin{array}{l}\mathrm{Fe}_{\mathrm{ox} 6} / \\
\mathrm{Fe}_{\mathrm{dit}}\end{array}$ & $\begin{array}{l}\mathbf{F e}_{\text {ox } 3} / \\
\mathbf{F e}_{\text {dit }}\end{array}$ & $\mathrm{Mn}_{\mathrm{ox} 6}$ & $\mathrm{Mn}_{\text {ox } 3}$ & $\mathbf{M n}_{\mathrm{dit}}$ & $\begin{array}{l}M n_{\text {ox } 6 /} \\
M n_{\text {dit }}\end{array}$ & $\begin{array}{l}M n_{\text {ox } 3 /} \\
M n_{\text {dit }}\end{array}$ \\
\hline & \multicolumn{3}{|c|}{$\mathrm{g} \mathrm{kg}^{-1}$} & & & \multicolumn{3}{|c|}{$\mathrm{g} \mathrm{kg}^{-1}$} & & \\
\hline Planossolo hidromórfico eutrófico solódico & 0,37 & 1,9 & 5,5 & 0,07 & 0,35 & 0,12 & 0,20 & 1,65 & 0,07 & 0,12 \\
\hline Gleissolo háplico Ta distrófico & 0,28 & 1,6 & 4,8 & 0,06 & 0,33 & 0,09 & 0,30 & 1,80 & 0,05 & 0,17 \\
\hline Planossolo hidromórfico eutrófico arênico & 0,19 & 1,1 & 3,1 & 0,06 & 0,35 & 0,18 & 0,30 & 2,00 & 0,09 & 0,15 \\
\hline rúptico & 0,92 & 4,9 & 8 , & 0, & 0,61 & 0,3 & 0,8 & 2,2 & 0,1 & 0,36 \\
\hline Vertissolo e & 1,35 & 6,7 & 10,7 & 0,13 & 0,63 & 0,49 & 1,20 & 2,20 & 0,22 & 0,55 \\
\hline Chernossolo ebânico carbonático vértico & 1,45 & 7,7 & 10,8 & 0,13 & 0,63 & 0,70 & 1,90 & 2,60 & 0,27 & 0,73 \\
\hline
\end{tabular}

(ox6): Extração com oxalato de amônio a pH6; (ox3): Extração com oxalato de amônio a pH3; e (dit): extração com ditionitocitrato-bicarbonato.

$\mathrm{Fe}_{\text {dit }}$, em relação aos demais. A razão $\mathrm{Fe}_{\mathrm{ox} 6} / \mathrm{Fe}_{\text {dit }}$ apresentou a maior associação com a CMAP em todos os solos utilizados (Quadro 4). Syers et al. (1971), trabalhando com solos Latossolos e Nitossolos oriundos de basaltos do Rio Grande do Sul com elevados conteúdos de óxidos de $\mathrm{Fe}$, encontraram associação da CMAP com as formas de $\mathrm{Fe}$ e com a razão $\mathrm{Fe}_{\mathrm{Ox}} /$ $\mathrm{Fe}_{\text {dit }}$. Resultados semelhantes foram observados por Hernandez \& Meurer (1998), trabalhando com as seguintes classes de solo (EUA, 1992): Typic Argiudoll Colônia Palma, Typic Hapludert Itapebi, Typic Argiudoll Algorta, Typic Argiudoll Arroyo Blanco, Typic Argiudoll S.Gabriel-Guaycuru e Typic Hapludert Kiyú. Esses solos são utilizados em sistemas mistos arroz-pastagens. A área superficial específica elevada, presente nos óxidos de baixa cristalinidade, resulta em maior adsorção de $\mathrm{P}$, em comparação com os óxidos cristalinos.

Os solos oriundos de sedimento de basalto apresentaram também maiores teores totais de $\mathrm{Mn}$ quando comparados aos solos oriundos de sedimento de arenito e granito (Quadro 2). Entre esses solos, o Chernossolo apresentou maiores teores de $\mathrm{Mn}_{\text {dit }} \mathrm{e}$ $\mathrm{Mn}_{\text {ox }}$ e maior razão $\mathrm{Mn}_{\mathrm{Ox} 6} / \mathrm{Mn}_{\text {dit. }}$. É provável que a maior CMAP nos solos oriundos de sedimento de basalto possa ser também atribuído aos maiores teores totais de $\mathrm{Mn}$ e razões $\mathrm{Mn}_{\text {Ox6 }} / \mathrm{Mn}_{\text {dit }}$ em relação aos solos oriundos de sedimento de granito (Shahandeh et al., 2003).

Quadro 3. Coeficiente linear (b0), coeficiente angular (b1) e coeficiente de determinação $\left(R^{2}\right)$ da relação linear entre ( $\mathrm{P}$ sol/P ads) x Psol e a capacidade máxima de adsorção de fósforo (b) em seis solos orizícolas do Rio Grande do Sul

\begin{tabular}{lcccc}
\hline \multicolumn{1}{c}{ Solo } & b0 & b1 & $\mathbf{R}^{2}$ & b \\
\hline & & & & $\mathrm{mg} \mathrm{kg}^{-1}$ \\
Planossolo (SGe 3) & 0,2389 & $0,0076^{*}$ & $0,93^{* *}$ & 132 \\
Gleissolo & 0,0842 & $0,0101^{*}$ & $0,97^{* *}$ & 99 \\
Planossolo (SGe 1) & 0,8908 & $0,0137^{*}$ & $0,97^{* *}$ & 73 \\
Luvissolo & 0,0078 & $0,0022^{*}$ & $0,97^{* *}$ & 455 \\
Vertissolo & 0,0059 & $0,0018^{*}$ & $0,99^{* *}$ & 556 \\
Chernossolo & 0,0056 & $0,0017^{*}$ & $0,94^{* *}$ & 588 \\
\hline * e $^{* *}$ : significativos a 5 e 1\%, respectivamente.
\end{tabular}

${ }^{*} \mathrm{e}^{* *}$ : significativos a 5 e $1 \%$, respectivamente.
Quadro 4. Coeficientes de correlação linear simples (R) entre a CMAP e as variáveis: teores absolutos das formas de Fe e de Mn e relações entre os óxidos de baixa cristalinidade e os cristalinos nas amostras dos solos: Planossolo (SGe 3); Gleissolo, Planossolo (SGe 1); Luvissolo; Vertissolo; e Chernossolo

\begin{tabular}{|c|c|}
\hline Variável & $\mathbf{R}$ \\
\hline $\mathrm{CMAP}_{\mathrm{x}} \mathrm{Fe}_{\mathrm{Ox} 6}$ & $0,98^{* *}$ \\
\hline CMAP $\times \mathrm{Fe}_{\mathrm{O} \times 3}$ & $0,98^{* *}$ \\
\hline CMAP $\times \mathrm{Fe}_{\mathrm{dit}}$ & $0,95^{* *}$ \\
\hline $\mathrm{CMAP} \times \mathrm{Fe}_{\mathrm{Ox} 6} / \mathrm{Fe}_{\text {dit }}$ & $0,99 * *$ \\
\hline $\mathrm{CMAP}_{\mathrm{X} \mathrm{Fe}} \mathrm{Ox} / \mathrm{Fe}_{\text {dit }}$ & $0,97^{* *}$ \\
\hline CMAP x Mn Ox6 & $0,87^{* *}$ \\
\hline CMAP x Mn Ox3 & $0,84^{* *}$ \\
\hline CMAP x Mn dit & $0,85^{* *}$ \\
\hline $\mathrm{CMAP} \times \mathrm{Mn}_{\mathrm{Ox} 6} / \mathrm{Mn}_{\mathrm{dit}}$ & $0,90 * *$ \\
\hline CMAP x Mn ${ }_{0 \times 3} / \mathrm{Mn}_{\text {dit }}$ & $0,88^{* *}$ \\
\hline CMAP x Argila & $0,70^{*}$ \\
\hline CMAP x Mał́ria Orgânica & $0,56^{\mathrm{ns}}$ \\
\hline
\end{tabular}

* $\mathrm{e}^{* *}$ : significativos a 5 e $1 \% \mathrm{e}^{\mathrm{Ns}}$ : não significativo, respectivamente.

Outra característica do solo que está relacionada à adsorção de $\mathrm{P}$ é a fração argila. Neste estudo, essa característica apresentou correlação significativa com a CMAP (Quadro 4). Isso indica que, à medida que ocorre aumento na quantidade de argila, os solos apresentam maior adsorção de $\mathrm{P}$. Isso se deve à presença predominante de óxidos de $\mathrm{Fe}$ na fração argila dos solos utilizados. Os íons $\mathrm{OH}^{-}$e $\mathrm{OH}_{2}$ presentes na superfície dos óxidos de Fe são deslocados pelos ânions fosfato para a solução do solo (Sample et al., 1980).

A matéria orgânica apresentou correlação positiva não significativa com a CMAP. Segundo Pavan \& Myazawa (1983), esse atributo pode aumentar a fração de óxidos de Fe de baixa cristalinidade, resultando em maior capacidade de adsorção de $\mathrm{P}$.

Os dados obtidos no experimento de sorção de $\mathrm{P}$ em solos reduzidos indicaram que os solos utilizados apresentaram comportamentos distintos e estavam associados aos materiais de origem, principalmente com o comportamento dos óxidos de Fe em ambiente de oxidação e redução do solo. 
Quadro 5. Teor de P sorvido, Fe, pH e Mn em amostras dos solos Planossolo, Luvissolo, Vertissolo e Chernossolo submetidas a 5, 15, 30 e 45 dias de alagamento

\begin{tabular}{|c|c|c|c|c|}
\hline \multirow{2}{*}{ Solo } & \multicolumn{4}{|c|}{ Alagamento (dia) } \\
\hline & 5 & 15 & 30 & 45 \\
\hline & \multicolumn{4}{|c|}{$\mathrm{P}$ sorvido, $\mathrm{mg} \mathrm{kg}^{-1}$} \\
\hline Planossolo & $162 \mathrm{Da}$ & $154 \mathrm{Db}$ & $157 \mathrm{Cb}$ & $163 \mathrm{Ca}$ \\
\hline Luvissolo & $168 \mathrm{Cb}$ & $171 \mathrm{Cab}$ & $169 \mathrm{Bab}$ & $172 \mathrm{Ba}$ \\
\hline Vertissolo & $173 \mathrm{Ba}$ & $176 \mathrm{Ba}$ & $175 \mathrm{Aa}$ & $176 \mathrm{Aa}$ \\
\hline \multirow[t]{2}{*}{ Chernossolo } & $177 \mathrm{Aa}$ & $180 \mathrm{Aa}$ & $178 \mathrm{Aa}$ & $179 \mathrm{Aa}$ \\
\hline & \multicolumn{4}{|c|}{$\mathrm{Fe}, \mathrm{mg} \mathrm{L}^{-1}$} \\
\hline Planossolo & $30 \mathrm{Ac}$ & $60 \mathrm{Ab}$ & $90 \mathrm{Aa}$ & $83 \mathrm{Aa}$ \\
\hline Luvissolo & $13 \mathrm{Bc}$ & $21 \mathrm{Bb}$ & $58 \mathrm{Bb}$ & $85 \mathrm{Aa}$ \\
\hline Vertissolo & $5 \mathrm{Cc}$ & $8 \mathrm{Cc}$ & $29 \mathrm{Cb}$ & $68 \mathrm{Ba}$ \\
\hline \multirow[t]{2}{*}{ Chernossolo } & $4,5 \mathrm{Cc}$ & $7,2 \mathrm{Cc}$ & $32 \mathrm{Cb}$ & $70 \mathrm{Ba}$ \\
\hline & \multicolumn{4}{|c|}{$\mathrm{pH}$} \\
\hline Planossolo & $5,85 \mathrm{Ac}$ & $6,28 \mathrm{Ab}$ & $6,30 \mathrm{Ab}$ & $6,45 \mathrm{Aa}$ \\
\hline Luvissolo & $5,62 \mathrm{Bc}$ & $5,80 \mathrm{Bb}$ & $6,14 \mathrm{Aa}$ & $6,22 \mathrm{ABa}$ \\
\hline Vertissolo & $5,40 \mathrm{Cd}$ & $5,65 \mathrm{BCc}$ & $5,88 \mathrm{Bb}$ & $6,12 \mathrm{Ba}$ \\
\hline \multirow[t]{2}{*}{ Chernossolo } & $5,28 \mathrm{Cd}$ & $5,60 \mathrm{Cc}$ & $5,93 \mathrm{Bb}$ & $6,15 \mathrm{Ba}$ \\
\hline & \multicolumn{4}{|c|}{$\mathrm{Mn}, \mathrm{mg} \mathrm{L}^{-1}$} \\
\hline Planossolo & $2,58 \mathrm{Ca}$ & $2,55 \mathrm{Ba}$ & $2,58 \mathrm{Ba}$ & $2,58 \mathrm{Ca}$ \\
\hline Luvissolo & $2,73 \mathrm{BCb}$ & $7,53 \mathrm{Aa}$ & $7,68 \mathrm{Aa}$ & $7,55 \mathrm{Ba}$ \\
\hline Vertissolo & $2,83 \mathrm{Bb}$ & 7,68 Aa & $7,82 \mathrm{Aa}$ & $7,73 \mathrm{ABa}$ \\
\hline Chernossolo & $3,45 \mathrm{Ab}$ & $7,75 \mathrm{Aa}$ & $7,80 \mathrm{Aa}$ & $7,85 \mathrm{Aa}$ \\
\hline
\end{tabular}

Médias seguidas de letras maiúsculas distintas nas colunas e minúsculas nas linhas diferem estatisticamente pelo teste de Duncan a $5 \%$.

O solo Planossolo apresentou menor sorção de $\mathrm{P}$ do que os solos oriundos de sedimento de basalto em todos os períodos de alagamento. Isso se deve à maior intensidade de redução dos óxidos férricos a óxidos ferrosos, com liberação do $\mathrm{P}$ adsorvido, observada no Planossolo, principalmente no período de 5 a 30 dias de alagamento. A partir dos 45 dias de alagamento, o aumento da sorção de $\mathrm{P}$ quando comparado aos 30 dias de alagamento foi proporcionado, provavelmente, pela diminuição da solubilidade dos óxidos ferrosos, devido ao valor de $\mathrm{pH}$ atingido nesse período, resultando na formação de $\mathrm{Fe}(\mathrm{OH})_{3}$, os quais adsorvem $\mathrm{P}$ na solução do solo.

Os solos Vertissolo e Chernossolo não apresentaram diferenças significativas na quantidade de $\mathrm{P}$ sorvido entre os períodos de alagamento avaliados. A maior redução dos óxidos férricos a ferrosos aos 30 e 45 dias de alagamento em relação aos períodos anteriores não foi suficiente para que existisse diferença significativa na sorção de P.

O solo Luvissolo apresentou comportamento intermediário entre os demais solos oriundos de basalto e o solo Planossolo.

A influência do Mn na sorção e dessorção em solos reduzidos não foi constatada nos solos utilizados. No Planossolo, houve estabilização nos teores de Mn na solução ao longo do período de alagamento. Logo, as oscilações na sorção de P nesse solo provavelmente não estiveram associadas à dinâmica do Mn. Nos solos oriundos de sedimento de basalto (Luvissolo,
Vertissolo e Chernossolo) ocorreu maior liberação de Mn a partir dos 15 dias de alagamento. Entretanto, essa diferença não promoveu alterações na quantidade de $\mathrm{P}$ sorvido, indicando que não há associação do aumento da solubilidade do Mn com a dessorção de P.

\section{CONCLUSÕES}

1. Os solos oriundos de sedimento de basalto apresentaram maiores teores totais de $\mathrm{Fe}$ e $\mathrm{Mn}$ e também razões de $\mathrm{Fe}_{\text {ox } 6} / \mathrm{Fe}_{\text {dit }}$ e $\mathrm{Mn}_{\mathrm{ox} 6} / \mathrm{Mn}_{\text {dit }}$, proporcionando maior CMAP do que os solos derivados de sedimento de arenito e granito.

2. A CMAP foi mais associada com a razão $\mathrm{Fe}_{\mathrm{Ox} 6} /$ $\mathrm{Fe}_{\text {dit }}$ do que $\mathrm{Mn}_{\mathrm{Ox} 6} / \mathrm{Mn}_{\text {dit }}$.

3. O solo Planossolo apresentou menor sorção de P do que os solos oriundos de sedimento de basalto em todos os períodos de alagamento.

\section{LITERATURA CITADA}

EMBRAPA BRASILEIRA DE PESQUISA AGROPECUÁRIA EMBRAPA. Centro Nacional de Pesquisa de Solos. Sistema brasileiro de classificação de solos. 2.ed. Rio de Janeiro, Embrapa Solos, 2006. 306p. 
ESTADOS UNIDOS. Department of Agriculture. Soil Survey Staff. Keys to soil taxonomy, 5. Blaksburg, Pocahontas, 1992. 56p. (SMSS Technical Monograph, 19)

GONÇALVES, G.K.; SOUSA, R.O.; VAHL, L.C. \& BORTOLON, L. Solubilização dos fosfatos naturais Pato de Minas e Arad em dois solos alagados. R. Bras. Ci. Solo, $32: 2157-2164,2008$.

HERNANDEZ, J. \& MEURER, E.J. Adsorção de fósforo e sua relação com formas de ferro em dez solos do Uruguai. R. Bras. Ci. Solo, 22:223-230, 1998.

INDA JUNIOR, A.V. \& KÄMPF, N. Avaliação de procedimentos de extração dos óxidos de ferro pedogênicos com ditionitocitrato-bicarbonato de sódio. R. Bras. Ci. Solo, 27:1139 $1147,2003$.

KHALID, R.A.; PATRICK JR, W.H. \& DELAUNE, R.D. Phosphorus sorption characteristics of flooded soils. Soil Sci. Soc. Am. J., 41:305-309, 1977.

MEHRA, O.P. \& JACKSON, M.L. Iron oxide removal from soils and clay by a dithionite-citrate system buffered with sodium bicarbonate. Clays Clay Miner., 7:317-327, 1960.

McKENZIE, R.M. Manganese oxides and hydroxides. In: DIXON, J.B. \& WEED, S.B., eds. Minerals in soil environments. Madison, Soil Science Society of America, 1989. p.439-465.

MURPHY, J. \& RILLEY, J.P. A modified single solution method for the determination of phosphate in natural waters. Anal. Chem. Acta, 27:31-36, 1962.

PAVAN, M.A. \& MIYAZAWA, M. Química dos solos inundados. Londrina, Iapar, 1983. Separata de treinamento em arroz irrigado e alternativas agrícolas em várzeas. Londrina, Iapar, 1983. p.5-20.

PONNAMPERUMA, F.N. Chemical kinetics of wetland rice soils relative to soil fertlity. In: INTERNATIONAL RICE RESEARCH INSTITUTE. Wetland soils: Characterization, classification, and utilization. Los Baños, 1978. p.71-90.

SAH, R.N.; MIKKELSEN, D.S. \& HAFEZ, A.A. Phosphorus behavior in flooded-drained soils. I. Effects on phosphorus sorption. Soil Sci. Soc. Am. J., 53:1718-1722, 1989.

SAMPLE, E.C.; SOPER, R.J. \& RACZ, G.J. Reactions of phosphate fertilizers in soils. In: KHASAWNEH, F.E.; SAMPLE, E.C. \& KAMPRATH, E.J., eds. The role of phosphorus in agriculture. Madison, American Society of Agronomy, 1980. p.263-310.
SCHWERTMANN, U. Differenzierung der eisenoxide des bodens durch extraction mit ammoniumoxalat-losung. Zeitschrift Pflanzenernahrung Bod., 105:194-202, 1964.

SCHWERTMANN, U. Inhibitory effect of soil organic matter on the crystallization of amorphous ferric hydroxide. Nature, 212:645-646, 1966.

SCHWERTMANN, U. \& TAYLOR, R.M. Iron oxides. In: DIXON, J.B. \& WEED, S.B., eds. Minerals in soil environments. Madison, Soil Science Society of America, 1989. p.379-438.

SHAHANDEH, H.; HOSSNER, L.R. \& TURNER, F.T. Phosphorus relationships to manganese and iron in rice soils. Soil Sci., 168:489-500, 2003.

SOUSA, R.O. Oxirredução em solos alagados afetada por resíduos vegetais. Porto Alegre, Universidade Federal do Rio Grande do Sul, 2001. 139p. (Tese de Mestrado).

SYERS, J.K.; EVANS, T.D.; WILLIAMS, J.D.H. \& MURDOCK, J.T. Phosphate sorption parameters of representative soils from Rio Grande do Sul, Brazil. Soil Sci., 112:267$275,1971$.

TEDESCO, M. J.; GIANELLO, C.; BISSANI, C.A.; BOHNEN, H. \& VOLKWEISS, S. J. Análises de solo, plantas e outros materiais. 2 ed. Porto Alegre: Departamento de solos da UFRGS, 1995. 174p. (Boletim Técnico de Solos, 5)

VAHL, L.C.; ANGHINONI, I. \& KAMPF, N. Previsão da acumulação de ferro trocável no solo durante o alagamento através de análise química. In: CONGRESSO BRASILEIRO DE CIÊNCIA DO SOLO, 24., Goiânia, 1993. Resumos... Goiânia, 1993. p.183-184.

VAHL, L.C.; GUIDOTTI, R.M.M. \& FABRES, R.T. Análise química de solo para a estimativa da acumulação de $\mathrm{Fe}^{2+}$ e $\mathrm{Mn}^{2+}$ durante o alagamento. In: CONGRESSO BRASILEIRO DE ARROZ IRRIGADO, 1., Pelotas, 1999. Anais... Pelotas, Embrapa Clima Temperado, 1999.

ZONTA, E.P.; MACHADO, A.A. \& SILVEIRA, J.P. Sistema de análise estatística para microcomputadores(SANEST). Pelotas, Universidade Federal de Pelotas, 1984. 151p. 
